\title{
HP1 $\gamma$ function is required for male germ cell survival and spermatogenesis
}

\author{
Jeremy P Brown'1, Jörn Bullwinkel'1, Bettina Baron-Lühr'1, Mustafa Billur'1, Philipp Schneider ${ }^{1}$, Heinz Winking² and \\ Prim B Singh*1
}

\begin{abstract}
Background: HP1 proteins are conserved components of eukaryotic constitutive heterochromatin. In mammals, there are three genes that encode HP1-like proteins, termed HP1a, HP1 $\beta$ and HP1 $\gamma$, which have a high degree of homology This paper describes for the first time, to our knowledge, the physiological function of HP1 $\gamma$ using a gene-targeted mouse.

Results: While targeting the Cbx3 gene (encoding the HP1 1 protein) with a conditional targeting vector, we generated a hypomorphic allele (Cbx3hypo), which resulted in much reduced (barely detectable) levels of HP1Y protein. Homozygotes for the hypomorphic allele (Cbx3hypo/hypo) are rare, with only $1 \%$ of Cbx3hypo/hypo animals reaching adulthood. Adult males exhibit a severe hypogonadism that is associated with a loss of germ cells, with some seminiferous tubules retaining only the supporting Sertoli cells (Sertoli cell-only phenotype). The percentage of seminiferous tubules that are positive for L1 ORF1 protein (ORF1p) in Cbx3hypo/hypo testes is greater than that for wildtype testes, indicating that L1 retrotransposon silencing is reversed, leading to ectopic expression of ORF1p in Cbx3hypo/ hypo germ cells.

Conclusions: The Cbx3 gene product (the HP1 1 p protein) has a non-redundant function during spermatogenesis that cannot be compensated for by the other two HP1 isotypes. The Cbx3hypo/hypo spermatogenesis defect is similar to that found in Miwi2 and Dnmt3L mutants. The Cbx3 gene-targeted mice generated in this study provide an appropriate model for the study of HP1 $y$ in transposon silencing and parental imprinting.
\end{abstract}

\section{Background}

The presence of methylated lysine 9 of histone H3 (H3K9ME) and structural heterochromatin protein (HP) 1 proteins are characteristic evolutionarily conserved hallmarks of heterochromatin [1]. In mammals, there are three HP1 isotypes, which have a high degree of homology, termed HP1 $\alpha$ (encoded by the Cbx5 gene), HP $1 \beta$ (encoded by the $C b x 1$ gene) and HP1Y (encoded by the $C b x 3$ gene) [2,3]. Despite the significant degree of sequence conservation shared between the mammalian HP1 isotypes, several studies have indicated that they are likely to have non-redundant functions. First, their nuclear localization patterns are different: $\mathrm{HP} 1 \alpha$ and HP $1 \beta$ are usually found enriched at sites of constitutive heterochromatin, whereas HP1 $\gamma$ has a more uniform dis-

* Correspondence: psingh@fz-borstel.de

1 Division of Immunoepigenetics, Department of Immunology and Cell Biology, Research Center Borstel, D-23845 Borstel, Germany

Full list of author information is available at the end of the article tribution [4-6]. Second, biochemical assays have identified isotype-specific binding partners [7] and, third, targeted deletion of the $C b \times 1$ gene has shown that it is essential, and that its loss of function cannot be compensated for by the products of the $C b \times 5$ and $C b x 3$ genes [8].

Analysis of the $C b x 1$ null mutant has shown that the $C b x 1$ gene product, HP1 $\beta$, is required for proper development of the brain, with $C b \times 1^{-1-}$ neurospheres cultured in vitro showing a dramatic genomic instability that is indicative of a defect in centromere function [8]. Interestingly, the lethality of the $C b \times 1$ mutation compared with the observed viability of the $\operatorname{Suv}(3) 9 h 1 / h 2$ histone methyl transferase (HMTase) double mutant [9] shows that the essential function(s) of HP1 $\beta$ lies outside its interaction with the heterochromatic H3K9ME3 determinant generated by the $\operatorname{Suv}(3) 9 \mathrm{~h} 1 / \mathrm{h} 2$ HMTases [3]. By contrast, homozygous $\mathrm{Cb} \times 5^{-1}$ mutants are indistinguishable from wild-type littermates, indicating that its function is 
redundant [8] (Singh PB: unpublished data). To date, nothing is known about the biological function of the $\mathrm{Cb} \times 3$ gene.

The mouse $C b \times 3$ gene lies on chromosome 6 , and is tightly linked to the Hnrnpa2b1 gene [10]. Both genes, which are divergently transcribed, share a $3 \mathrm{~kb} C \mathrm{CpG}$ island that is conserved in the syntenic HNRNPA2B1$C B X 3$ region in humans [11]. Fragments from the CpGrich HNRNPA2B1-CBX3 region have been shown to be able to confer high-level expression of linked transgenes in the mouse and thus, it has been termed a ubiquitously acting chromatin opening element (UCOE) $[12,13]$.

In a first attempt to elucidate the biological function of the $C b x 3$ gene, we undertook a gene-targeting experiment using a conditional targeting vector. During production of this conditional mutation, we fortuitously generated a hypomorphic allele of $C b \times 3\left(C b \times 3^{h y p o}\right)$, which results in a dramatic reduction in HP1 1 protein expression to barely detectable levels; expression of the Hnrnpa2b1 protein was not affected. The number of Cbx3 hypo/hypo homozygotes that survive to adulthood is low, with adult males exhibiting a severe spermatogenic defect. This result confirms the non-redundant functions of mammalian HP1 proteins, and provides the first insight into the function of HP1Y during development. We also observed a dramatic reduction in the number of

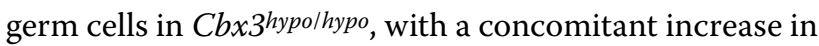
expression of the ORF1 protein encoded by the LINE-1 (L1) retrotransposon. These data indicate that HP1 $\gamma$ might be part of a Miwi2-HP1Y silencing pathway that is required for proper germ-cell renewal and survival in the testes.

\section{Results and discussion}

The conditional targeting vector used to disrupt the $C b x 3$ gene was designed with a FlpE-excisable neo-tk cassette placed in the intron separating exons 3 and 4 (Figure 1, second and third rows). Disruption of $C b x 3$ gene function, after excision of the neo-tk by FlpE, would be effected by $\mathrm{Cre}$ excision of exons 2 and 3, which contain the alternative ATG start codons of the $C b x 3$ gene (Figure 1 , third and fourth rows). Targeting frequency with the neo-tk-containing conditional vector (Figure 1, second row) was approximately 1:100. Germline transmission of the targeted allele (Figure 1, third row) was achieved, and intercrossing of the heterozygotes for the targeted allele resulted in a normal mendelian ratio of wild-type to targeted alleles at E19 (not shown) the day before birth. However, the number of adult mice that were homozygous for the targeted allele was low, with only 3 of 216 adult males and 1 of 193 adult females being homozygous for the targeted allele (Figure 1, third row), which was designated $C b x 3^{\text {hypo }}$. The nature and timing of the attrition of Cbx3hypolhypo homozygotes that takes place after birth is not known, and the data presented below on $C b \times 3^{\text {hypoolhypo }}$ adult animals are based on the three males and one female that reached adulthood.

The greatly reduced numbers of $C b x 3^{\text {hypo } / \text { hypo adults }}$ prompted us to investigate whether the targeting event itself had affected $C b \times 3$ expression, and if this was the likely cause of the reduced numbers of Cbx3hypolhypo adults. To explore this hypothesis further, we generated primary mouse fibroblasts from E13.5 wild-type and Cbx3hypo/hypo littermates and compared the expression levels of HP1 1 by Western blotting. As shown in Figure 2, there was a dramatic reduction in HP1 $1 \gamma$ expression levels in $C b x 3$ hypo/hypo compared with wild-type mouse embryonic fibroblasts (MEFs) (Figure 2a, top row: wild-type to hypo/hypo) indicating that the $C b x 3^{\text {hypo }}$ allele was a hypomorph. The effect of the targeting event was specific to the $C b x 3$ gene, as protein expression of the closely linked Hnrnpa $2 b 1$ gene was not changed (Figure 2a, middle row). Given this unexpected result, we were prompted to investigate whether the presence of the neo-tk selection cassette itself was interfering with $C b x 3$ expression. Previous work has shown that knockdown of target gene expression can result from the presence of a neo gene in the targeting vector [14]. The mechanism for such a knockdown is not fully understood but may involve transcriptional interference, by which the presence of one transcriptional unit interferes with another that is in cis [15].

To test the hypothesis that the neo-tk selection cassette was interfering with $C b x 3$ expression, we took advantage of the fact that neo-tk cassette is flanked by FRT sites that allow its excision by FlpE expression (Figure 1). When the $n e o-t k$ cassette was excised after electroporation of FlpE mRNA into Cbx $3^{\text {hypo } / \text { hypo }} \mathrm{MEFs}$, the HP1 $\gamma$ expression levels returned to normal wild-type levels (Figure 2a, top row: FlpE treated) indicating that it was indeed the presence of the neo-tk cassette that resulted in the reduced HP1 $y$ levels. As a control, the $C b x 3$ gene was disrupted by Cre expression, resulting in $C b x 3^{-/-}$MEFs and complete loss of HP1Y expression (Figure 2, top row: -/-). The Western blot analysis was complemented with immunofluorescence experiments, which confirmed that the presence of the neo-tk cassette affected $C b x 3$ expression (Figure $2 \mathrm{~b}$ ). The reduced levels of $\mathrm{HP} 1 \gamma$ protein in Cbx3 hypo/hypo MEFs did not affect the expression of HP $1 \alpha$ and HP1 $\beta$ as measured by immunofluorescence and Western blot analysis (see Additional files 1 and 2, Figures s1 and s2). Western blot analysis also revealed that there were no significant changes between $C b x 3^{\text {hypo } / \text { hypo }}$ and wild-type MEFs in the levels of three different histone post-translational modifications, H3K9ME3, H4K20ME3 and H3K9AC (see Additional file 2, Figure s2). When $\mathrm{Cbx}^{-1-}$ MEFs were included into this analysis, 


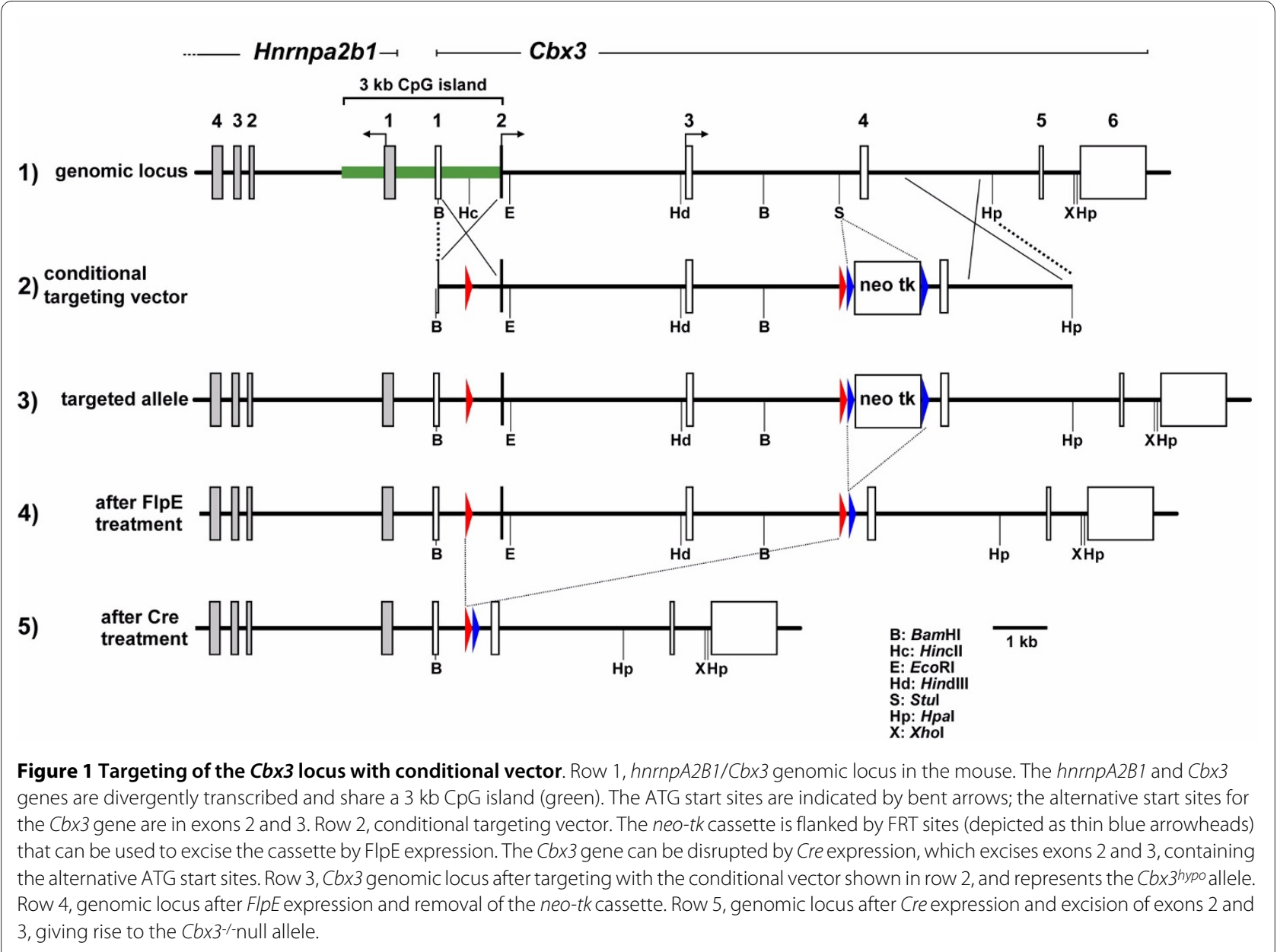

we observed an increase in H4K20me3 levels compared with wild-type and $C b x 3^{\text {hypo } h y p o} \mathrm{MEFs}$ (see Additional file 2, Figure s2), indicating that complete loss of HP1y in Cbx $3^{-1-c e l l s ~ m i g h t ~ a f f e c t ~ t h e ~ a c t i v i t y ~ o f ~ e n z y m e s ~ i n v o l v e d ~}$ in regulating this determinant of the histone code.

A dramatic reduction of $\mathrm{HP} 1 \gamma$ levels was also observed in the Cbx $3^{\text {hypo/hypo }}$ mouse tissues; Western blot analysis revealed that HP1Y levels were reduced to almost undetectable levels in all tissues examined (Figure 3). There

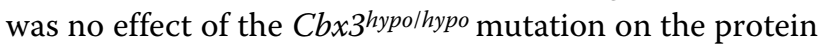
levels of HP1 $\alpha$ (see Additional file 3, Figure s3a) and HP1 $\beta$ (see Additional file 3, Figure s3b) or on the same three histone post-translational modifications H4K20ME3, H3K9ME3 and H3K9AC (see Additional file 3 , Figures s3c to s3e).

Housing the three $C b x 3^{\text {hypo } h y p o}$ males with wild-type females resulted in no litters, indicating a possible problem with male fertility. The males were killed and the testes removed, which revealed severe hypogonadism compared with wild-type males (Additional file 4, Figure s4). Examination of wild-type testes showed that expression of the $C b x 3$ gene product, HP1 $\gamma$, was present in vir- tually all cells (Figure 4a) and is distinct from the staining pattern of transcriptional intermediary factor (TIF) $1 \beta$, a $\mathrm{HP} 1 \gamma$-interacting protein, in wild-type testes, where preleptotene and spermatogonia are TIF1 $\beta$-negative [16]. Sertoli cells are prominently stained, as are round (stage 2-6) spermatids (Figure $4 \mathrm{~b}$ ), with the latter showing an enriched spot of HP1 $\gamma$ staining in the nucleus (Figure $4 \mathrm{~b}$; see also Additional file 5 , Figure $\mathrm{s} 5 \mathrm{~b}$ ), which probably represents the characteristic heterochromatic chromocenter found in these nuclei [17]. HP1 $\gamma$ is excluded from meiotic metaphase chromosomes (Figure 4c), which is similar to the known expulsion of the bulk of HP1 proteins from metaphase chromosomes by the so-called serine 10 phosphorylation switch [18]. It may well be that the chromosome condensation during meiosis or mitosis requires the removal of the bulk of HP1 proteins from the chromosomes. In pachytene spermatocytes, $\mathrm{HP} 1 \gamma$ is found throughout the nucleus but is enriched at a few sites that probably represent heterochromatic chromocenters (Figures $4 \mathrm{~d}$; see also Additional file 5, figure s5a). HP1 $\gamma$ staining of spermatogenic cell types is detectable until elongating spermatid stage 10 (Figure $4 \mathrm{~d}$; Table 1), which 


\section{A}

HP1 $\gamma$

hnrnpa2b1

$\beta$-Actin wt
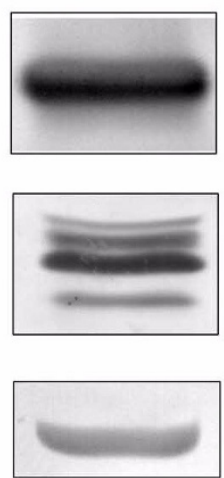

hypo/hypo
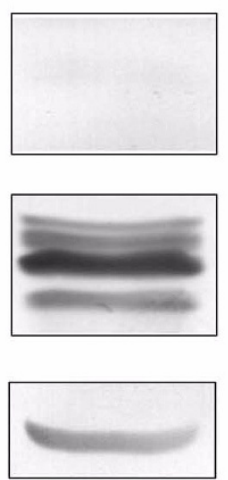

$\%$

FlpE treated (Cre treated)
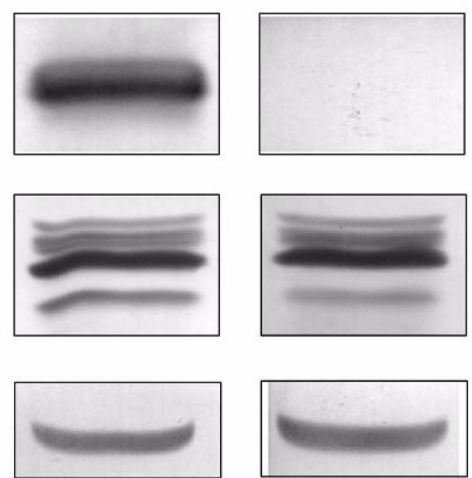

B
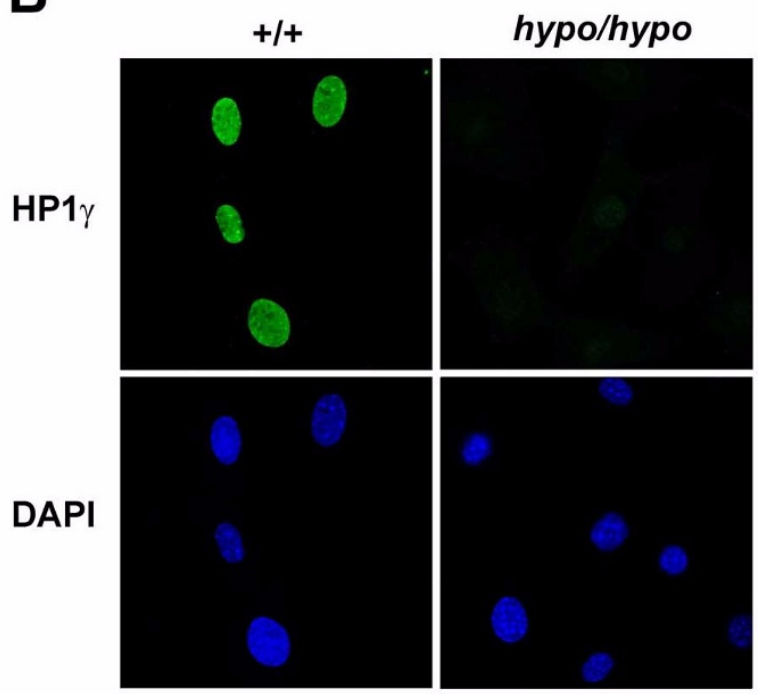

\section{FIpE treated}

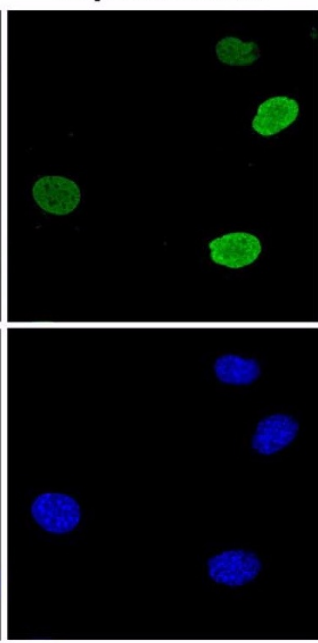

- (Cre treated)
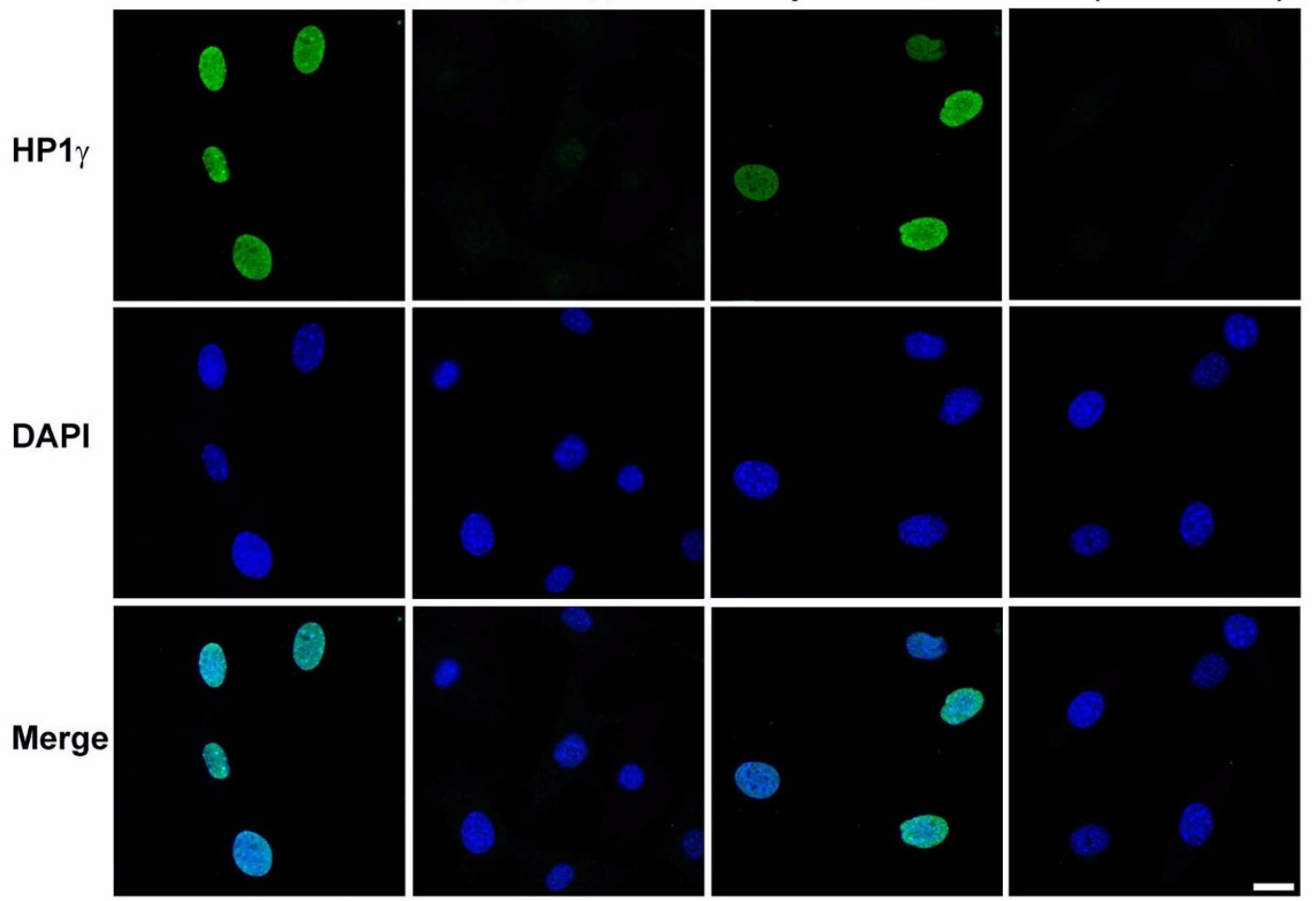

Figure 2 Presence of the neo-tk cassette results in a hypomorphic (Cbx3hypo) allele. (a) Row 1, expression of HP1 $\gamma$ in MEFs. HP1 $\gamma$ expression was dramatically reduced to almost undetectable levels in MEFs derived from embryos that are homozygous for the targeted allele shown in row 3 of Figure 1 (Cbx3hypo/hypo MEFs). After expression of FlpE in the Cbx3hypo/hypo MEFs (FlpE-treated MEFs), the expression of HP1 1 returned to wild-type levels. HP1 1 p protein expression in the FlpE-treated MEFs could be extinguished by expression of Cre recombinase, giving rise to Cbx3\% MEFs. Hnrnpa2b1 protein expression was not affected by the presence or absence of the neo-tk cassette, as shown in row 2. Row 3, actin loading control. (b) Row 1 , immunofluorescent HP1 $\gamma$ staining of MEFs. The typical punctate HP1 $\gamma$ pattern of expression in wild-type MEFs was reduced to very faint, almost background, levels in Cbx3hypo/hypo MEFs. Strikingly, HP1 $y$ staining levels returned to wild-type levels after the neo-tk cassette was excised after Flpe treatment (FlpE-treated MEFs). HP1 $\gamma$ staining was lost after expression of Cre recombinase giving rise to Cbx3-- MEFs. Row 2, DAPI staining; row 3, merged images. Bar $=20 \mu \mathrm{m}$. 


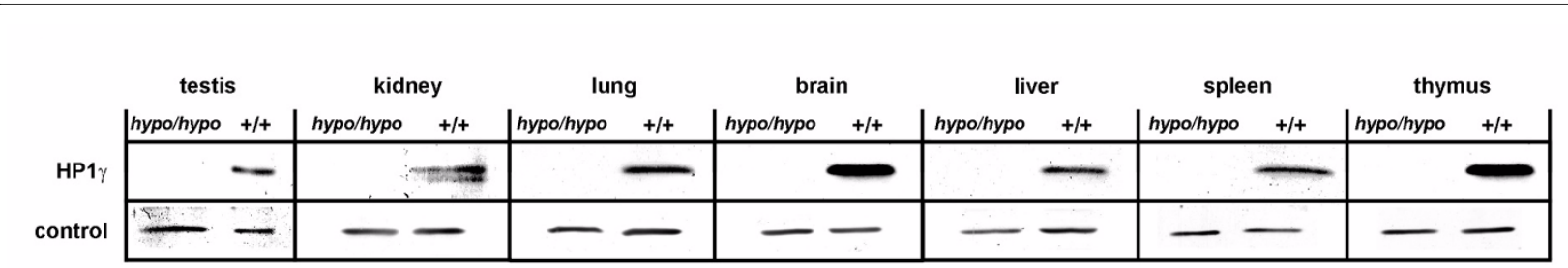

Figure $3 \mathrm{HP} 1 y$ protein expression was dramatically reduced in Cbx3hypo/hypo tissues. Protein expression was reduced to almost undetectable levels in testis, kidney, lung, brain, liver spleen and thymus tissues from the Cbx3hypo/hypomice.

is around the time that the bulk of the histones are removed and replaced by the protamines [19].

By contrast, HP1 $\gamma$ staining was almost undetectable in sections taken from Cbx3hypo/hypo testes (Figure 4e, f). Histological examination revealed a severe impairment of spermatogenesis in Cbx3hypo/hypo testes. The diameter of the tubules in Cbx3hypo/hypo testes was smaller (0.13 $\mathrm{mm})$ than that in wild-type testes $(0.2 \mathrm{~mm})$. Of 70 tubules examined, 22 were almost completely devoid of germ cells (for example, Figure 4e, upper right tubule) and 48 tubules had impaired spermatogenesis. Tubules in which mature sperm could be observed were rare (Figure 4f, especially the magnified inset). The loss of germ cells was confirmed using a germ-specific antibody (anti-germ cell nuclear antigen (GCNA)) [20], which revealed a dramatic reduction in germ cells (GCNA-positive cells) in Cbx3hypo/hypo testes (Figure 5c, Figure 5d) compared with wild-type mice (Figure 5, Figure 5b). Some of the tubules in Cbx3hypo/hypo testes exhibited a Sertoli cellonly (SCO) phenotype, reminiscent of the tubules seen in the Dnmt3L and Miwi2 mutants [21,22]. Thus, the Cbx3hypo/hypo mutation results in the general suppression of spermatogenesis, which can vary from tubule to tubule; in some tubules suppression is complete, resulting in a SCO phenotype, whereas in others, spermatogenesis can proceed and give rise to some mature sperms. This variation across the tubules might reflect the fact that the Cbx3hypo mutation is 'leaky', that is, the interference of $\mathrm{Cbx} 3$ by the neo-tk is variable giving rise to leaky expression of Cbx3. Some Cbx3hypo/hypo germ cells and their progeny might have closer to wild-type levels of $\mathrm{HP} 1 \gamma$, thus enabling greater likelihood of survival with more normal development.

The similarity of the Cbx $3_{\text {hypo/hypo }}$ spermatogenesis defect to Dnmt3L and Miwi2 mutants [21,22] prompted us to investigate whether there were any changes in the expression of retrotransposon expression in the mutant testes. For this, we used a polyclonal antibody to the L1encoded ORF1 protein [23]. ORF1p is required for L1 transposition, and its levels of expression are increased in germ cells, as the L1 transposons become de-repressed $[24,25]$. Using this antibody, we found that $45 \%$ of the tubules in Cbx3hypo/hypotestes that contained germ cells were positive by immunohistochemistry for ORF1 protein expression, compared with $5 \%$ in wild-type testes (see Additional file 6 and 7, Figure s6 and Figure s7). Again, this indicates that the $C b x 3^{\text {hypolhypo }}$ mutation may affect the same silencing pathway that is affected in the Dnmt3L and Miwi2 mutants [21,22].

We next investigated whether the $C b x 3^{\text {hypo }}$ mutation affects the expression of the other two HP1 isotypes, HP $1 \alpha$ and HP $1 \beta$. Accordingly, we stained wild-type and

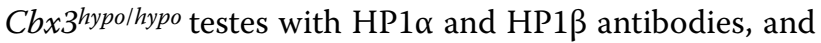
compared the cell types and levels of staining for the two proteins on the different genetic backgrounds. For $H P 1 \alpha$, most cells of the wild-type testes were HP1 $\alpha$-positive (see Additional file 8, Figure s8). Sertoli cells were stained with anti-HP1 $\alpha$ (see Additional file 8, Figure s8, black arrows) as were pachytene spermatocytes, where HP1 $\alpha$ was enriched within a few bright foci which probably represent heterochromatic regions (see Additional file 8, Figure $s 8 \mathrm{~b}$, blue arrows). The round (stage 2-6) spermatids

Table 1: Spermatid stages at which HP1a, HP1 $\beta$ and HP1 1 protein expression was extinguished.

\begin{tabular}{|c|c|c|c|c|c|}
\hline \multirow[t]{2}{*}{ Protein } & \multicolumn{5}{|c|}{ Spermatid stage number } \\
\hline & 7 & 8 & 9 & 10 & 11 \\
\hline HP1a & + & - & - & - & - \\
\hline HP1 $\beta$ & + & + & + & + & - \\
\hline HP1y & + & + & + & + & - \\
\hline
\end{tabular}




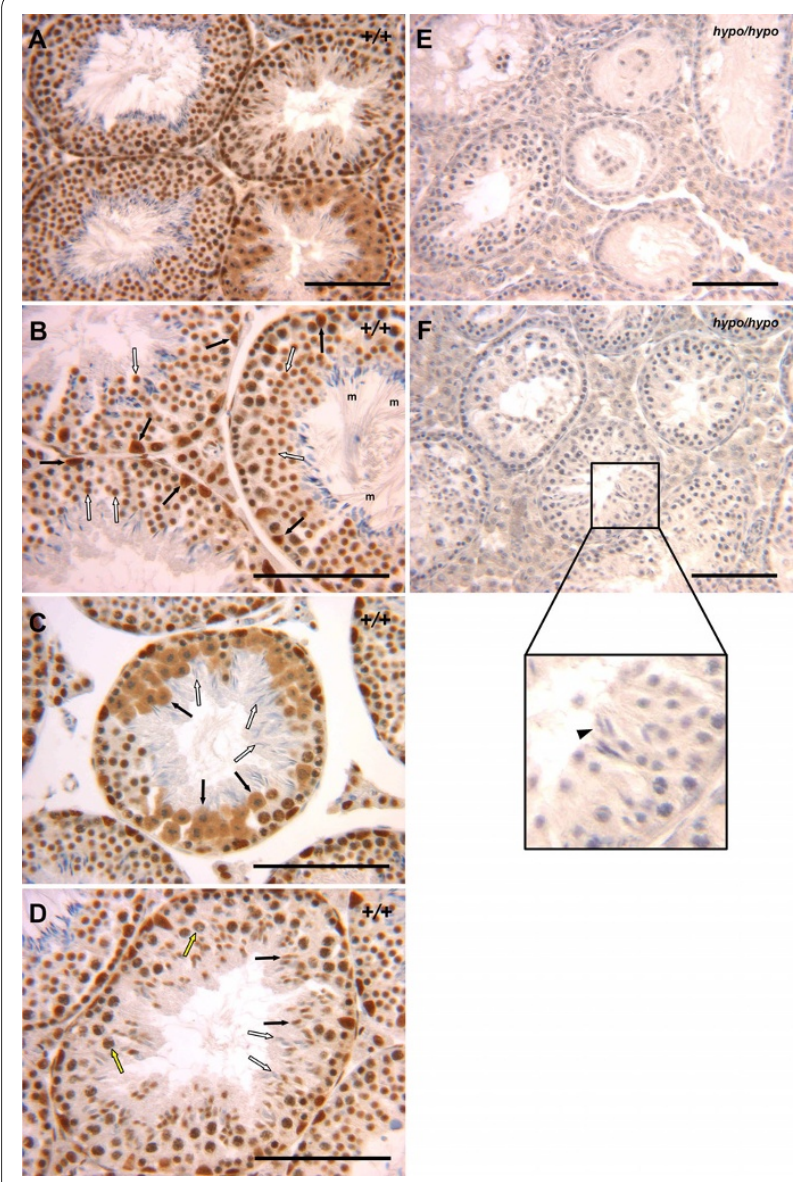

Figure $4 \mathrm{Cbx} 3^{\text {hypo/hypo }}$ testes had severe impairment of spermatogenesis. (a) HP1 1 protein was found in nearly all cells of the wild-type testes. (b) In wild-type tubules, Sertoli cells stained strongly for HP1Y (black arrows) as did round spermatids (stage 2-6) (white arrows). Many round spermatid nuclei possessed an enriched focus of HP1 1 staining that was characteristic of the single block of heterochromatin observed at this stage (see Additional file 5, Figures 5b). (c) HP1 $y$ was largely excluded from meiotic metaphase chromosomes and was found surrounding the condensed chromosomes in the meiotic cytoplasm (black arrows). Mature sperm (white arrows) were negative for HP1Y. (d) Stage 10 spermatids, at around the time they elongated, were either positive (black arrows) or negative (white arrows) for HP1 1 , indicating that it was during this stage that levels of HP1 1 proteins decrease. Pachytene spermatocytes (yellow arrows) showed a few brightly stained spots that represent sites of constitutive heterochromatin (see Additional file 5, Figures 5a). (e) In Cbx3hypo/hypo testes, HP1Y staining was very weak and the tubules showed severely impaired spermatogenesis with greatly reduced numbers of cells and some tubules exhibiting a Sertoli cells-only phenotype (see upper right tubule). (f) In some tubules of Cbx3hypo/hypo testes, mature sperm could be observed (inset, black arrowhead). Bar $=100 \mu \mathrm{m}$.

were also stained and exhibited a single spot of staining in the nucleus, which is characteristic of the heterochromatic chromocenter found in these cell types (see Additional file 8, Figure s8c, white arrows). Meiotic chromosomes were not stained but, unlike HP1 $\gamma$ staining in wild-type testes, very little staining was observed in the

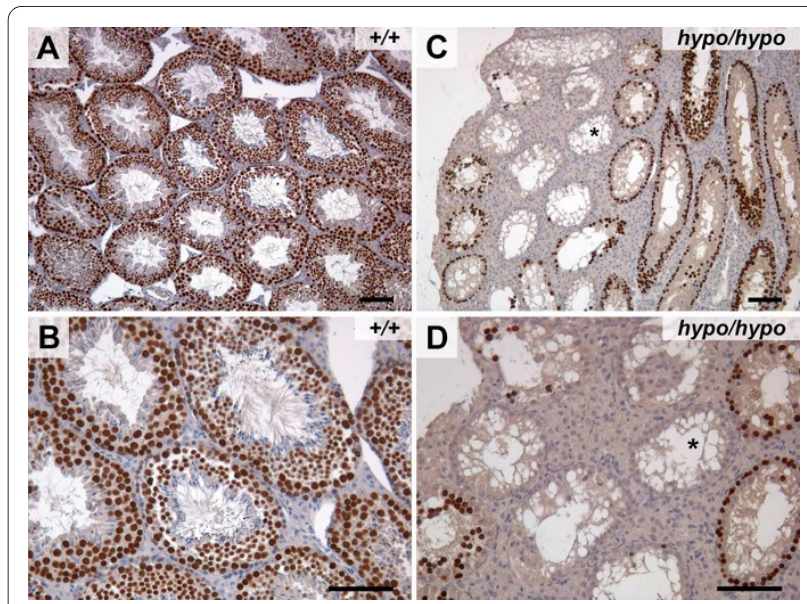

Figure $5 \mathrm{Cb} \times 3^{\text {hypo/hypo }}$ testes showed a dramatic loss in the number of germ cells. $(\mathbf{a}, \mathbf{b})$ Typical germ cell nuclear antigen (GCNA)

staining of germ cells within wild-type testes. $(\mathbf{c}, \mathbf{d})$ In Cbx3hypo/hypo testes, there was a dramatic reduction in the numbers of GCNA-positive germ cells. Some tubules contained no GCNA-positive germ cells and presented a Sertolin cells-only phenotype (asterisk). Bar $=100 \mu \mathrm{m}$.

meiotic cytoplasm (see Additional file 8, Figure s8c, arrowheads). In addition, unlike with HP1 $\gamma$, there are some cells, probably spermatogonia, which were not stained by the anti-HP1 $\alpha$ antibody (see in Additional file 8 , Figure $\mathrm{s} 8 \mathrm{~b}$, yellow arrows). In wild-type testes, HP $1 \alpha$ staining was lost at an earlier stage than HP1y staining, with stage 7 spermatids (see Additional file 8, Figure s8b, arrowheads) being the last stage at which HP1 $\alpha$ was still seen (Table 1). In the Cbx3hypo/hypo testes, the cell types stained were the same as those found in wild-type cells, notwithstanding the obvious suppression of spermatogenesis seen in the $C b x 3^{\text {hypoolhypo }}$ testes (see Additional file 8 , Figure s8d, Figure s8e). The levels of $\mathrm{HP} 1 \alpha$ staining

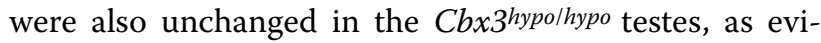
denced by the typical staining of the round (stage 2-6) spermatids in Cbx3hypo/hypo testes (see Additional file 8, Figure s8e, white arrows). HP1 $\beta$ staining of wild-type testes (see Additional file 9, Figure s9) was similar to that for HP1 $\alpha$ (see Additional file 8, Figure s8), with the only difference being that the staining of HP $1 \beta$ was still visible at a later stage, in stage 10 spermatids, as was seen for HP1y (Table 1) [26]. The levels of HP1 $\beta$ staining and the cell types stained in Cbx3hypo/hypo testes (see Additional file 9, Figure s9d, Figure s9e) were not significantly different to those in the wild-type testes. These data indicate that the defects seen in the Cbx $3^{\text {hypo/hypo }}$ mutation are unlikely to operate through changes in the expression of the other two isotypes, HP1 $\alpha$ and HP1 $\beta$.

The clear differences between wild-type and Cbx3hypol hypoadult testes were not observed in embryonic E17 testes. The morphology of the seminiferous tubules and numbers of gonocytes in wild-type and Cbx3hypo/hypo tes- 
tes were similar (see Additional file 10, Figure s10), indicating that the suppression of spermatogenesis seen in

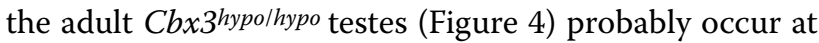
later stages, after meiosis has been initiated.

Housing the one adult Cbx3 3 ypo/hypo female mouse with wild-type males also resulted in no litters. Although it is difficult to conclude from a single Cbx3 hypo/hypo animal

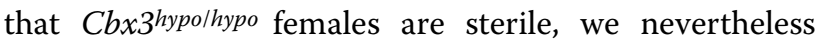
decided to examine sections of wild-type and Cbx3 $3^{\text {hypo }}$ hypo ovaries. Examination of the sections revealed no obvious morphological difference between wild-type and Cbx3 $3^{\text {hypo/hypo }}$ ovaries; all stages of folliculogenesis were observed in Cbx3hypo/hypo ovaries, including corpora lutea, indicating that ovulation was normal in the Cbx3 hypo/hypo female (data not shown).

The similarity of the Cbx3 hypo/hypo phenotype in the testes with those observed in the testes of Miwi2 [21] and Dnmt3L mutants [21] is suggestive. Both Miwi2 and Dnmt3L are involved in DNA methylation of interspersed repeats during spermatogenesis, and mutation of either Miwi2 or Dnmt3L results in a SCO phenotype and the loss of DNA methylation of transposons, resulting in their ectopic expression [21,22]. Our analysis of the $C b x 3^{h y p o}$ mutation indicates that $\mathrm{HP} 1 \gamma$ might also be involved in a Miwi2-HP1 silencing pathway, as observed for HP1a-PIWI pathway in Drosophila [27]. These data, in conjunction with the generation of a Cre-inducible $C b \times 3$ allele from the $C b x 3^{\text {hypo }}$ allele (unpublished), form a sound basis for investigating the role of $\mathrm{HP} 1 \gamma$ in transposon silencing and parental imprinting.

\section{Conclusion}

$\mathrm{HP} 1 \gamma$ has a non-redundant function that cannot be rescued by the other HP1 isotypes, HP1 $\alpha$ and HP1 $\beta$. This function is essential for male germ cell survival and proper spermatogenesis.

\section{Methods}

\section{Animal studies}

The experimental research on mice was carried out in accordance with German animal protection law, and the study has been approved by the Ministerium für Landwirtschaft, Umwelt und ländliche Räume of SchleswigHolstein in Kiel (Germany).

\section{Staining of testes sections}

Testes were fixed in Bouin's fixative (saturated aqueous solution of picric acid, 37\% formaldehyde, and glacial acetic acid, 15:5:1) overnight, embedded in paraffin wax and cut into sections $2 \mu \mathrm{m}$ thick. Subsequent antigen retrieval by pressure cooker and indirect immunoperoxidase staining was performed as described previously [28]. In addition, blocking solution (Image-iT FX Signal Enhancer; Invitrogen, Carlsbad, CA, USA) was applied for 30 minutes to reduce background staining. All antibodies were diluted in Tris-buffered saline with 10\% bovine serum albumin (BSA). Endogenous peroxidase was inactivated with $3 \% \mathrm{H}_{2} \mathrm{O}_{2}$, and diaminobenzidine (DAB; Sigma, St. Louis, MO, USA) was used to detect peroxidase activity. Primary antibodies used in this study were anti-GCNA1 (mAB 10D9G11, kind gift of Professor G C Enders), anti-HP1 $\alpha$, anti-HP1 $\beta$ [4] and anti-HP1 $\gamma$ (all Chemicon, Temecula, CA, USA). Species-specific horseradish-peroxidase coupled secondary antibodies were purchased from Dianova (Hamburg, Germany). Images were photographed with a microscope and camera (DMLB2 microscope and DFC320 camera; Leica, Basel, Switzerland).

For L1 ORF1p staining, paraffin wax-embedded sections were dewaxed and subsequently incubated for 15 minutes with $1 \%$ peroxide followed by 15 minutes with signal enhancer (Image-iT FX Signal Enhancer; Invitrogen). For antigen retrieval, pancreatic trypsin $(1 \mathrm{mg} / \mathrm{ml}$ in phosphate-buffered saline (PBS)) was applied for 2 minutes. The samples were incubated with anti-mouse ORF1p antibody (kind gift of Professor S L Martin) at 1:500 dilution in PBS with $10 \%$ BSA overnight at $4^{\circ} \mathrm{C}$, with secondary antibody and peroxidase detection with DAB performed as described above. Finally, the sections were incubated with haematoxylin for 6 minutes. Images were taken with a Olympus (Hamburg, Germany) DS-Ri1 microscope, an Nikon (Melville, NY, USA) BX41 camera and NIS-Elements documentation software. Tubules were scored negative for ORF1p if the resident germ cells exhibited haematoxylin staining only with no brown staining (see Additional file 6, Figure s6c, Figure s6e). Tubules that were scored positive for ORF1p contained germ cells with robust brown staining of the nucleus and cytoplasm (see Additional file 6, Figure 6c, Figure s6e). Cbx3 hypo/hypo tubules that had no germ cells (see Additional file 6, Figure s6b, asterisks) were not included in the analysis.

\section{Western blotting}

Western blotting was performed essentially as described previously [8]. For histone isolation, tissues were cut to pieces and further disintegrated in buffer $\mathrm{A}(10 \mathrm{mM}$ HEPES, pH 7.9, $1.5 \mathrm{mM} \mathrm{MgCl}_{2}, 10 \mathrm{mM} \mathrm{KCl}, 0.5 \mathrm{mM}$ dithiothreitol (DTT) and Complete Mini EDTA-free Protease Inhibitor (Roche, Mannheim, Germany)) with a Dounce homogenizer (50 strokes with a tight pestle). Nuclei were pelleted and then resuspended in buffer S1 (0.25 $\mathrm{M}$ sucrose, $10 \mathrm{mM} \mathrm{MgCl}_{2}$ and protease inhibitor) and layered over an equal volume of buffer S3 (0.88 M saccharose, $0.5 \mathrm{mM} \mathrm{MgCl}_{2}$ and protease inhibitor). After separation by centrifugation $(2,800 \boldsymbol{g}$ for 10 minutes at $\left.4^{\circ} \mathrm{C}\right)$ the pellet was resuspended in extraction buffer $(1 \mathrm{M}$ 
$\mathrm{HCl}, 0.02 \% \beta-\beta$ mercaptoethanol and protease inhibitor) and incubated at $4^{\circ} \mathrm{C}$ overnight. Pellet was extracted twice. The supernatants were pooled and treated with 10 volumes of acetone for precipitation $\left(-20^{\circ} \mathrm{C}\right.$ overnight). After separation by centrifugation $(10,000 \mathrm{~g} g$ for $4 \mathrm{~min}$ utes at $\left.4^{\circ} \mathrm{C}\right)$, the pellet was reconstituted in water and finally denatured in Laemmli buffer $\left(5\right.$ minutes at $\left.95^{\circ} \mathrm{C}\right)$ for SDS-PAGE.

For Western blotting, primary antibodies for $H P 1 \alpha$, HP1 $\beta$, HP1 $\gamma$, histone 3, histone 4, H3K9AC (all Chemicon), $\beta$-Actin (Sigma), hnRNP A2B1 (Biozol, Eching, Germany), H3K9ME3 [29] and H4K20ME3 [30] were used. Detection of these antibodies was carried out either with colorimetric reaction through species-specific AP-coupled secondary antibodies (Dianova, Germany) or by incubation with infrared dye-coupled secondary antibodies (Alexa Fluor 680-coupled goat anti-mouse (Invitrogen) and IRDye $800 \mathrm{CW}$ anti-rabbit (LI-COR, Lincoln, NE, USA)) and subsequent scanning with an infrared imager (Odyssey; LI-COR).

\section{Immunofluorescence}

Immunofluorescence was performed as described previously [31]. Primary antibodies used in this study were against HP1 $\alpha, \mathrm{HP} 1 \beta$ and $\mathrm{HP} 1 \gamma$ (all Chemicon). Nucleic acids were stained with 4',6-diamidino-2-phenylindole (1 $\mu \mathrm{g} / \mathrm{ml})$. Confocal images were acquired, processed and assembled as described previously [8].

\section{Additional material}

Additional file 1 Figure s1. Immunofluorescent staining of HP1a and HP1 $\beta$ was not changed in wild-type and Cbx3 mutant backgrounds. (a) There was no significant difference in HP1 a staining on wild-type and Cbx3 mutant backgrounds (row 1, green). Row 2, staining with 4',6-diamidino-2phenylindole (DAPI) of cells depicted in the panels above. Images of rows 1 and 2 were merged in row 3. (b) There was no significant difference in HP1 $\beta$ staining on wild-type and Cbx3 mutant backgrounds (row 1, green). Row 2, DAPI staining of cells depicted in the panels above. Images of rows 1 and 2 were merged in row 3. Bar $=20 \mu \mathrm{m}$.

Additional file $\mathbf{2}$ Figure s2. There was no significant difference in the levels of HP1a, HP1 3, H3K9ME3, H3K9AC in wild-type MEFs compared with

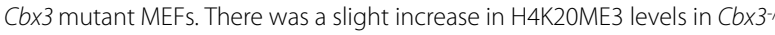
- MEFs compared with wild-type and Cbx3hypo/hypo MEFs.

Additional file $\mathbf{3}$ Figure $\mathbf{s} \mathbf{3}$. There was no significant difference in the levels of HP1a, HP1ß, H3K9ME3, H4K20ME3, H3K9AC in Cbx3hypo/hypo extracts taken from testis, kidney, lung, brain, liver, spleen and thymus tissues compared with extracts for the corresponding wild-type tissues. ND = not detectable.

Additional file 4 Figure s4. Testes from Cbx3 $3^{\text {hypo/hypo }}$ males exhibited severe hypogonodism. Bar $=50 \mathrm{~mm}$.

Additional file $\mathbf{5}$ Figure s5. (a) Many pachytene spermatocytes (black arrows) from wild-type animals had punctuate staining, with the intensely staining areas probably representing regions of heterochromatin (see inset). (b) Many round spermatids from wild-type animals exhibited a 'spot' that was enriched for HP1y staining and probably represents the single block of heterochromatin that was characteristically found in these cells.
Additional file $\mathbf{6}$ Figure $\mathbf{s} 6$. LINE-1 (L1) retrotransposon expression was increased in Cbx3 $3^{\text {hypo/hypo }}$ compared with wild-type testes, as shown by an increase in germ cells staining positive for the L1-encoded ORF1 protein (ORF1p). (a) A wild-type testis section stained with the anti-L1-encoded ORF1p antibody, showing regions from two tubules, one tubule negative for ORF1 $p$ and the other tubule positive for ORF1p, which are magnified in (c) and (d), respectively. (b) A Cbx3 hypo/hypo testis section stained with the anti-L1-encoded ORF1 $p$ antibody, showing regions from two tubules, one tubule negative for ORF1 $p$ and the other tubule positive for ORF1p, which are magnified in (e) and (f), respectively. The asterisks denote tubules that have the Sertoli cells-only phenotype and lack germ cells. (c) ORF1p-negative germ cells in a wild-type tubule. (d) ORF1p-positive germ cells in a wild-type tubule. (e) ORF1p-negative germ cells in a Cbx3 hypo/hypo tubule. (f) ORF1p-positive germ cells in a Cbx3hypo/hypotubule. Bar $=200 \mu \mathrm{m}$.

Additional file 7 Figure s7. In the Cbx3hypo/hypo testes section, $45 \%$ of the tubules contain ORF1p-positive germ cells compared with $5 \%$ of the tubules in the wild-type testes section.

Additional file 8 Figure $\mathbf{8 8}$. Distribution and levels of HP1a staining in tes tes was not affected by the Cbx3hypo/hypo mutation. (a) Most cells within the wild-type testes were stained with HP1a antibody. (b) A more detailed analysis of the HP1 a staining showed that Sertoli cells (black arrows) were clearly stained. Pachytene spermatocytes (blue arrows) exhibited punctuate staining, probably representing heterochromatin. HP1 a staining was lost in stage 7 round spermatids (black arrowhead), which was earlier than for HP1Y (see Figure 4; Table 1). In contrast to the HP1Y staining, there were some cells at the periphery of the tubule, probably spermatogonia (yellow arrows), which were not stained. (c) HP1a does not stain meiotic chromosomes (black arrowheads) and was present at low levels in the surrounding meiotic cytoplasm. Round spermatids (white arrows) possessed an enriched 'spot' of HP1 a staining. (d) In Cbx3 hypo/hypo testes, HP1 a stained nearly all cells as in the wild-type. (e) Round spermatids (white arrows) were stained and possessed the typical nuclear focus of staining that was typical for this cell type. Bar $=100 \mu \mathrm{m}$.

Additional file 9 Figure $\mathbf{s} 9$. Distribution and levels of HP1 $\beta$ staining in tes-

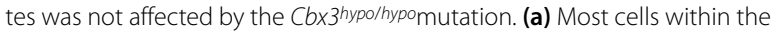
wild-type testes were stained with HP1 $\beta$-antibody. (b) A more detailed analysis of the HP1 $\beta$ staining showed that Sertoli cells (black arrowhead) were stained. HP1 $\beta$ did not stain meiotic chromosomes and was instead found in the meiotic cytoplasm (yellow arrows). There were also some cells at the periphery of the tubule, probably spermatogonia (black arrows), which were not stained. Inset shows pachytene spermatocytes with a punctuate pattern of staining (red arrows) probably heterochromatin. Round spermatids possessed an enriched 'spot' of HP1 $\beta$ staining (white arrows). (c) Expression of HP1 $\beta$ was lost at around the stage 10 spermatid stage. The box in (c) is magnified and two stage 10 spermatids are shown; one was still positive for HP1 $\beta$ (thick arrow, brown spermatid) the other was negative for HP1 $\beta$ (thin arrow; blue spermatid). (d) In Cbx3hypo/hypo testes, HP1 $\beta$ stained nearly all cells as in the wild-type. (E) In some tubules mature sperm (m) were visible. Bar $=100 \mu \mathrm{m}$.

Additional file $\mathbf{1 0}$ Figure s10. There was no difference in number of tubules and gonocytes between wild-type and Cbx3hypo/hypo E17 embryonic testes. (a) HP1 1 staining of E17 testes showed gonocytes heavily stained within the seminiferous tubules. (b) The HP1y staining of Cbx3hypo/hypo E17 embryonic testes was very weak although the number of gonocytes was not significantly less than wild type (tubules are outlined with a hatched line). Bar $=100 \mu \mathrm{m}$

\section{Competing interests}

The authors declare that they have no competing interests.

\section{Authors' contributions}

JPB made the conditional targeting vector and targeted ES cells, and helped to analyse the data. JB assembled all figures for manuscript and helped to analyse the data. MB and PS undertook staining of cells and Western blotting. BB-L undertook all the immunocytochemistry on testes sections. HW analysed the staining of testes. PBS conceived of and designed the study, and wrote the first draft of the paper. 


\section{Acknowledgements}

We thank Dmitris Kioussis and Ursula Menzel for production of chimeras from the targeted ES cells and Vladimir Shteyn for the hnrnpa2b1 blot. We thank Professors S L Martin and G C Enders for providing antibodies.

\section{Author Details}

'Division of Immunoepigenetics, Department of Immunology and Cell Biology, Research Center Borstel, D-23845 Borstel, Germany and 2Institut für Biologie, Universität Lübeck, D-23538 Lübeck, Germany

Received: 19 December 2009 Accepted: 27 April 2010 Published: 27 April 2010

\section{References}

1. Elgin SC, Grewal SI: Heterochromatin: silence is golden. Curr Biol 2003 13:895-898.

2. Jones DO, Cowell IG, Singh PB: Mammalian chromodomain proteins: their role in genome organisation and expression. Bioessays 2000, 22:124-137.

3. Billur M, Bartunik HD, Singh PB: The essential function of HP1beta: a case of the tail wagging the dog? Trends Biochem Sci 2010, 35:115-23.

4. Wreggett KA, Hill F, James PS, Hutchings A, Butcher GW, Singh PB: a mammalian homologue of Drosophila heterochromatin protein 1 (HP1) is a component of constitutive heterochromatin. Cytogenet Cell Genet 1994, 66:99-103.

5. Horsley D, Hutchings A, Butcher GW, Singh PB: M32, a murine homologue of Drosophila heterochromatin protein 1 (HP1), localises to euchromatin within interphase nuclei and is largely excluded from constitutive heterochromatin. Cytogenet Cell Genet 1996, 73:308-311.

6. Minc E, Allory Y, Worman HJ, Courvalin JC, Buendia B: Localization and phosphorylation of HP1 proteins during the cell cycle in mammalian cells. Chromosoma 1999, 108:220-234.

7. Kwon SH, Workman JL: The heterochromatin protein 1 (HP1) family: put away a bias toward HP1. Mol Cells 2008, 26:217-227.

8. Aucott R, Bullwinkel J, Yu Y, Shi W, Billur M, Brown JP, Menzel U, Kioussis D, Wang G, Reisert I, Weimer J, Pandita RK, Sharma GG, Pandita TK, Fundele R, Singh PB: HP1- $\beta$ is required for development of the cerebral neocortex and neuromuscular junctions. J Cell Biol 2008, 183:597-606.

9. Peters $A H, O^{\prime}$ Carroll D, Scherthan H, Mechtler K, Sauer S, Schöfer C Weipoltshammer K, Pagani M, Lachner M, Kohlmaier A, Opravil S, Doyle M, Sibilia M, Jenuwein T: Loss of the Suv39h histone methyltransferases impairs mammalian heterochromatin and genome stability. Cell 2001, 107:1323-337.

10. Sato M, Miyado K, Kimura M: Cloning and characterization of 5'upstream sequence of the M32 gene for a mouse homologue of Drosophila heterochromatin protein 1 (HP1). DNA Seq 2001, 12:97-106.

11. Williams S, Mustoe T, Mulcahy T, Griffiths M, Simpson D, Antoniou M, Irvine A, Mountain A, Crombie R: CpG-island fragments from the HNRPA2B1/CBX3 genomic locus reduce silencing and enhance transgene expression from the hCMV promoter/enhancer in mammalian cells. BMC Biotechnol 2005, 3:5-17.

12. Katsantoni EZ, Anghelescu NE, Rottier R, Moerland M, Antoniou M, de Crom R, Grosveld F, Strouboulis J: Ubiquitous expression of the rtTA2S$M 2$ inducible system in transgenic mice driven by the human hnRNPA2B1/CBX3 CpG island. BMC Dev Biol 2007, 7:108

13. Lindahl Allen M, Antoniou M: Correlation of DNA methylation with histone modifications across the HNRPA2B1-CBX3 ubiquitously-acting chromatin open element (UCOE). Epigenetics 2007, 2:227-236.

14. McDevitt MA, Shivdasani RA, Fujiwara Y, Yang H, Orkin SH: a "knockdown" mutation created by cis-element gene targeting reveals the dependence of erythroid cell maturation on the level of transcription factor GATA-1. Proc Nat/ Acad Sci USA 1997, 94:6781-6785.

15. Eszterhas SK, Bouhassira EE, Martin DI, Fiering S: Transcriptional interference by independently regulated genes occurs in any relative arrangement of the genes and is influenced by chromosomal integration position. Mol Cell Biol 2002, 22:469-479.

16. Weber P, Cammas F, Gerard C, Metzger D, Chambon C, Losson R, Mark M: Germ cell expression of the transcriptional co-repressor TIF1 $\beta$ is required for maintanence of spermatogeneis in the mouse. Development 2002, 129:2329-2337.
17. Govin J, Escoffier E, Rousseaux S, Kuhn L, Ferro M, Thévenon J, Catena R, Davidson I, Garin J, Khochbin S, Caron C: Pericentric heterochromatin reprogramming by new histone variants during mouse spermiogenesis. J Cell Biol 2007, 176:283-294.

18. Fischle W, Tseng BS, Dormann HL, Ueberheide BM, Garcia BA, Shabanowitz J, Hunt DF, Funabiki H, Allis CD: Regulation of HP1chromatin binding by histone $\mathrm{H} 3$ methylation and phosphorylation. Nature 2005, 438:1116-1122.

19. Zhao M, Shirley CR, Mounsey S, Meistrich ML: Nucleoprotein transitions during spermiogenesis in mice with transition nuclear protein Tnp1 and Tnp2 mutations. Biol Reprod 2004, 71:1016-1025.

20. Enders GC, May JJ: Developmentally regulated expression of a mouse germ cell nuclear antigen examined from embryonic day 11 to adult in male and female mice. Dev Biol 1994, 163:331-340.

21. Bourc'his D, Xu GL, Lin CS, Bollman B, Bestor TH: Dnmt3L and the establishment of maternal genomic imprints. Science 2001, 294:2536-2539.

22. Carmell MA, Girard A, Kant HJ van de, Bourc'his D, Bestor TH, de Rooij DG, Hannon GJ: MIWI2 is essential for spermatogenesis and repression of transposons in the mouse male germline. Dev Cell 2007, 12:503-514.

23. Martin SL: The ORF1 Protein encoded by LINE-1: structure and function during L1 retrotransposition. J Biomed Biotechno/ 2006, 2006:1-6.

24. Vagin W, Wohlschlegel J, Qu J, Jonsson Z, Huang X, Chuma S, Girard A, Sachidanandam R, Hannon GJ, Aravin AA: Proteomic analysis of murine Piwi proteins reveals a role for arginine methylation in specifying interaction with Tudor family members. Genes Dev 2009, 23:1749-62.

25. Soper SF, Heijden GW van der, Hardiman TC, Goodheart M, Martin SL, de Boer $P$, Bortvin A: Mouse maelstrom, a component of nuage, is essential for spermatogenesis and transposon repression in meiosis. Dev Cell 2008, 15:285-97

26. Hoyer-Fender S, Singh PB, Motzkus D: The murine heterochromatin protein M31 is associated with the chromocenter in round spermatids and Is a component of mature spermatozoa. Exp Cell Res 2000, 254:72-79.

27. Brower-Toland B, Findley SD, Jiang L, Liu L, Yin H, Dus M, Zhou P, Elgin SC, Lin H: Drosophila PIWI associates with chromatin and interacts directly with HP1a. Genes Dev 2007, 21:2300-2311.

28. Seitzer U, Hollmann C, Endl E, Gerdes J: Measuring immune responses in situ: immunofluorescent and immunoenzymatic techniques. In Methods in Microbiology Volume 32. 2nd edition. Edited by: Kaufmann SHE, Kabelitz D. Amsterdam: Academic Press; 2002:751-765.

29. Cowell IG, Aucott R, Mahadevaiah SK, Burgoyne PS, Huskisson N Bongiorni S, Prantera G, Fanti L, Pimpinelli S, Wu R, Gilbert DM, Shi W Fundele R, Morrison H, Jeppesen P, Singh PB: Heterochromatin, HP1 and methylation at lysine 9 of histone $\mathrm{H} 3$ in animals. Chromosoma 2002, 111:22-36.

30. Kourmouli N, Jeppesen P, Mahadevhaiah S, Burgoyne P, Wu R, Gilbert DM, Bongiorni S, Prantera G, Fanti L, Pimpinelli S, Shi W, Fundele R, Singh PB: Heterochromatin and tri-methylated lysine 20 of histone $\mathrm{H} 4$ in animals. J Cell Sci 2004, 117:2491-2501.

31. Scholzen T, Endl E, Wohlenberg C, Sar S van der, Cowell IG, Gerdes J, Singh PB: The Ki-67 protein interacts with members of the heterochromatin protein 1 (HP1) family: a potential role in the regulation of higher-order chromatin structure. J Pathol 2002, 196:135-144.

\section{doi: $10.1186 / 1756-8935-3-9$}

Cite this article as: Brown et al., HP1? function is required for male germ cell survival and spermatogenesis Epigenetics \& Chromatin 2010, 3:9 